\title{
Plantas medicinais com atividade repelente de mosquitos: protocolo de revisão sistemática
}

\author{
Medicinal plants with mosquito repellent activity: systematic review protocol \\ Plantas medicinales con actividad repelente de mosquitos: protocolo de revisión sistemática
}

Recebido: 08/10/2021 | Revisado: 13/10/2021 | Aceito: 13/10/2021 | Publicado: 16/10/2021

\author{
Anderson Ribeiro dos Santos \\ ORCID: https://orcid.org/0000-0003-3868-3380 \\ Universidade Federal de Sergipe, Brasil \\ E-mail: andersonfarmacia@yahoo.com.br \\ Alexia Mota Santos \\ ORCID: https://orcid.org/0000-0003-2715-6745 \\ Universidade Federal de Sergipe, Brasil \\ E-mail: alexiamotafarm@gmail.com \\ Fernando Henrique Oliveira de Almeida \\ ORCID: https://orcid.org/0000-0002-1728-9598 \\ Universidade Federal de Sergipe, Brasil \\ E-mail: fernandoalmeidafitofarm@gmail.com \\ Vinícius Freitas Arcieri de Medeiros \\ ORCID: https://orcid.org/0000-0003-0561-2420 \\ Universidade Federal de Sergipe, Brasil \\ E-mail: vfreitas12@gmail.com \\ Saulo Santos Matos \\ ORCID: https://orcid.org/0000-0002-6780-4236 \\ Universidade Federal de Sergipe, Brasil \\ E-mail: saulosm95@gmail.com \\ Thaís Feitoza de Carvalho \\ ORCID: https://orcid.org/0000-0001-7381-4791 \\ Universidade Federal de Sergipe, Brasil \\ E-mail: thaisfeitoza14@gmail.com \\ Carlos Adriano Santos Souza \\ ORCID: https://orcid.org/0000-0002-7913-2510 \\ Universidade Federal de Sergipe, Brasil \\ E-mail: carlos.953@gmail.com \\ Tamires Cardoso Lima \\ ORCID: https://orcid.org/0000-0001-6200-649X \\ Universidade Federal de Sergipe, Brasil \\ E-mail: tamires.cl87@gmail.com \\ Francilene Amaral da Silva \\ ORCID: https://orcid.org/0000-0001-6729-2843 \\ Universidade Federal de Sergipe, Brasil \\ E-mail: farmsilva@hotmail.com
}

\begin{abstract}
Resumo
Introdução: o uso de plantas medicinais com propriedades repelentes de mosquitos constitui uma alternativa eficaz, ecologicamente apropriada e menos agressiva ao meio ambiente. Objetivo: apresentar um protocolo de revisão sistemática de ensaios clínicos envolvendo plantas medicinais com atividade repelente de mosquitos. Métodos: o levantamento bibliográfico foi realizado nas bases de dados Science Direct, SciELO, SciFinder, Springer, PubMed/MEDLINE, Scopus e Web of Science. A busca foi realizada até agosto de 2021 e foram incluídos somente trabalhos publicados nas línguas portuguesa, inglesa ou espanhola. Os títulos, resumos e artigos na íntegra foram analisados por três revisores, de forma independente, para identificar os estudos relevantes. O referido protocolo foi registrado no International Prospective Register of Systematic Reviews (PROSPERO) e a revisão sistemática será conduzida de acordo com as diretrizes do Preferred Reporting Items for Systematic Reviews and Meta-Analyses (PRISMA). Considerações finais: a procura por novos repelentes derivados de plantas que sejam padronizados, eficazes e seguros na prevenção a doenças transmitidas por mosquitos torna relevante a realização dessa revisão sistemática.
\end{abstract}

Palavras-chave: Plantas medicinais; Óleos essenciais; Mosquitos; Atividade repelente; Protocolo; Revisão Sistemática. 


\begin{abstract}
Background: the use of medicinal plants with mosquito repellent properties constitutes an effective alternative, ecologically appropriate, and less aggressive to the environment. Objective: to present a protocol for a systematic review of clinical trials involving medicinal plants with mosquito repellent activity. Methods: the literature search was carried out using the Science Direct, SciELO, SciFinder, Springer, PubMed/MEDLINE, Scopus, and Web of Science databases. The search was carried out in August 2021 and included some work published in the Portuguese, English, or Spanish languages. The titles, abstracts, and articles in full were analyzed by three reviewers, independently, to identify the relevant studies. The referred protocol was registered in the International Prospective Register of Systematic Reviews (PROSPERO) and the systematic review will be conducted according to the guidelines for Preferred Reporting Items for Systematic Reviews and Meta-Analyzes (PRISMA). Final considerations: the search for new repellents derived from plants that are standardized, effective, and safe in the prevention of diseases transmitted by mosquitoes makes it relevant to carry out a systematic review.
\end{abstract}

Keywords: Medicinal plants; Essential oils; Mosquitoes; Repellent activity; Protocol; Systematic Review.

\title{
Resumen
}

Antecedentes: el uso de plantas medicinales con propiedades repelentes de mosquitos es una alternativa eficaz, ecológicamente apropiada y menos dañina para el medio ambiente. Objetivo: presentar un protocolo de revisión sistemática de ensayos clínicos con plantas medicinales con actividad repelente de mosquitos. Métodos: la encuesta bibliográfica se realizó en las bases de datos Science Direct, SciELO, SciFinder, Springer, PubMed/MEDLINE, Scopus y Web of Science. La búsqueda se realizó hasta agosto de 2021 y solo se incluyeron trabajos publicados en portugués, inglés o español. Tres revisores analizaron de forma independiente los títulos, resúmenes y artículos completos para identificar estudios relevantes. Este protocolo fue registrado en el International Prospective Register of Systematic Reviews (PROSPERO) y la revisión sistemática se llevará a cabo de acuerdo con las pautas de los Preferred Reporting Items for Systematic Reviews and Meta-Analyses (PRISMA). Consideraciones finales: la búsqueda de nuevos repelentes de origen vegetal estandarizados, efectivos y seguros en la prevención de enfermedades transmitidas por mosquitos hace que esta revisión sistemática sea relevante.

Palabras clave: Plantas medicinales; Aceites esenciales; Mosquitos; Actividad repelente; Protocolo; Revisión Sistemática.

\section{Introdução}

Os mosquitos são considerados um grave problema de saúde pública, pois além da irritação e desconforto que provocam durante a hematofagia, eles também atuam como vetores de importantes arboviroses para a saúde humana (Ali et al., 2012; Satyal et al., 2019). Existem, no mundo, aproximadamente 3.500 espécies de mosquitos conhecidas, sendo a maioria encontrada nas regiões tropicais e subtropicais. No entanto, desse total, somente $10 \%$ têm relevância médica e veterinária (Powel, 2018).

Os mosquitos que atuam como vetores de doenças humanas pertencem principalmente aos gêneros Aedes (chikungunya, vírus zika, dengue e febre amarela), Anopheles (malária e filariose) e Culex (encefalite japonesa, vírus do Nilo Ocidental e filariose) (Tandina et al., 2018). Em 2015, o vírus zika causou problemas em várias partes do mundo, incluindo Brasil, África, Ilhas do Pacífico e Sudeste Asiático. De acordo com a Organização Pan-Americana da Saúde (OPAS), mais de 40 países ou territórios nas Américas registraram epidemia de vírus zika entre 2015 e 2016, com mais de 500.000 casos suspeitos e confirmados de zika (Ali et al, 2017).

As estratégias atuais de controle de mosquitos baseiam-se no uso de inseticidas sintéticos, tais como o diclorodifeniltricloroetano (DDT), temefós, piretroides e malathion. Embora os agentes químicos tenham sido utilizados com sucesso para o controle de mosquitos durante muitas décadas, o seu uso contínuo e indiscriminado tem acarretado o desenvolvimento de resistência química, impactos ambientais e efeitos indesejáveis em organismos não-alvo, incluindo humanos. Nesse contexto, o uso de produtos naturais derivados de plantas para o controle de mosquitos vetores apresenta várias características atrativas, incluindo biodegradabilidade, ação e degradação rápidas, baixa toxicidade, baixa fitotoxicidade e maior seletividade (Ghosh et al., 2012; George et al., 2014; Wilke \& Marrelli, 2015).

Muitos essenciais obtidos de diferentes plantas aromáticas têm demonstrado potencial repelente, sendo eficazes contra diferentes espécies de mosquitos (Geetha \& Roy, 2014). Como exemplo, podemos destacar o óleo essencial de Cymbopogon 
nardus, conhecido popularmente como "citronela", um dos óleos essenciais mais comuns em patentes de repelentes de mosquitos (Pohlit et al., 2011). Considerando que o uso de repelentes à base de plantas medicinais constitui alternativa eficaz, ecologicamente apropriada e menos agressiva ao meio ambiente na prevenção às doenças causadas por mosquitos, o presente trabalha objetiva apresentar um protocolo de revisão sistemática de ensaios clínicos envolvendo plantas medicinais com atividade repelente de mosquitos.

O objetivo do referido trabalho é definir o caminho metodológico para elaboração e execução de uma Revisão Sistemática para avaliar a qualidade dos ensaios clínicos de plantas medicinais com atividade repelente contra mosquitos.

\section{Metodologia}

\subsection{Protocolo e Registro}

A revisão sistemática proposta será realizada com ensaios clínicos sobre a utilização plantas medicinais com propriedade repelentes de mosquitos. O protocolo desta revisão foi registrado no International Prospective Register of Systematic Reviews (PROSPERO), sob o número de registro CRD42017070923 (Anexo A). Ressalta-se que a revisão será conduzida de acordo com as diretrizes do Preferred Reporting Items for Systematic Reviews and Meta-Analyses (PRISMA). Esta declaração fornece informações essenciais sobre a metodologia e o desenvolvimento de revisões sistemáticas, tais como terminologia, formulação de questões de pesquisa, identificação e mineração de dados, qualidade do estudo, risco de viés na combinação dos dados (além do estudo seletivo) e viés nos resultados de publicação (Moher et al., 2015; Page et al., 2021).

\subsection{Estratégia de busca e fonte de dados}

O levantamento de dados para a revisão sistemática foi realizado nas bases de dados Science Direct, SciELO, SciFinder, Springer, PubMed/MEDLINE, Scopus e Web of Science. Além disso, também foi realizada uma busca manual por meio da análise das referências bibliográficas dos artigos incluídos. A busca foi realizada até agosto de 2021 e foram incluídos somente trabalhos publicados nas línguas portuguesa, inglesa ou espanhola.

Para realização da busca na literatura, serão utilizados os seguintes descritores do Medical Subject Headings (MeSH): "repelentes de insetos", "óleos essenciais", "extrato de plantas", "atividade repelente", "mosquitos" e "plantas". Os descritores serão adaptados de acordo com cada base de dados e combinados por meio dos operadores booleanos OR, AND e NOT. Os artigos selecionados e excluídos serão organizados utilizando o fluxograma PRISMA conforme apresentado na Figura 1. 
Figura 1 - Fluxograma para a seleção, exclusão, avaliação e inclusão dos estudos.
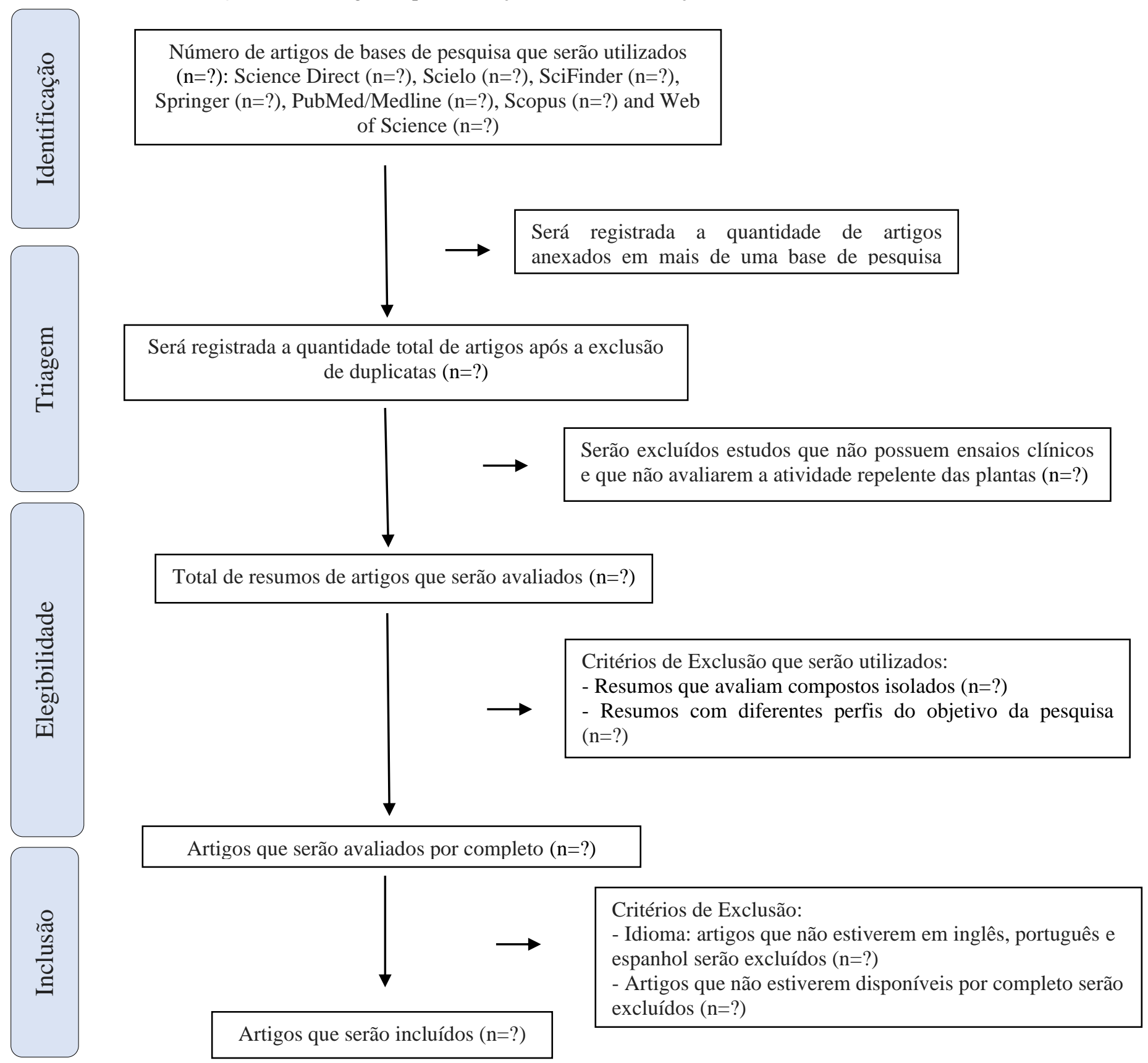

Fonte: Adaptado do PRISMA (2021).

\subsection{Seleção dos estudos}

Inicialmente, os registros da pesquisa serão importados para o software de gerenciamento de referência Rayyan (Ouzzani et al., 2016). A extração dos dados, a avaliação inicial dos registros relevantes seguido pelos resumos e artigos na íntegra será conduzida por dois revisores independentes (ARS e AMS). Em seguida, as divergências de seleção serão resolvidas por um terceiro revisor (CASS) para chegar em um consenso. Posteriormente, serão excluídos os artigos que não se enquadrarem no escopo desta revisão. A estatística Kappa de Cohen será utilizada para mensurar a confiabilidade entre os avaliadores (Mchugh, 2012).

As seguintes informações deverão extraídas dos estudos selecionados por meio de formulário padronizado: país em que o estudo foi realizado, data de publicação, duração do estudo, planta medicinal utilizada, espécie de mosquito utilizada nos 
ensaios, tempo de repelência, forma farmacêutica utilizada, padronização da forma farmacêutica utilizada, marcador químico utilizado na padronização, randomização, perdas e exclusões. O principal desfecho a ser avaliado é a eficácia da atividade repelente das plantas medicinais frente às espécies de mosquitos utilizados nos estudos.

\subsection{Análise e síntese de dados}

A qualidade metodológica (risco de viés) dos estudos será avaliada por meio da ferramenta de risco de viés da Cochrane (Cochrane Risk of Bias Tool) - ROB 2.0, disponível no Cochrane Handbook de revisões sistemáticas e intervenções em saúde (Higgins et al., 2011). Além disso, o GRADE (Grading of Recommendations Assessment Development and Evaluation) será usado para avaliar a qualidade da evidência (Brozek et al., 2009; Guyatt et al., 2009).

Dois programas de computador serão utilizados para realizar as análises e sínteses: Review Manager V.5.3 Copenhague: Nordic Cochrane Center, The Cochrane Collaboration, 2014 e o programa Microsoft Excel. Os estudos serão então julgados com um risco de viés baixo, incerto ou alto.

\section{Discussão}

A maioria das plantas medicinais com atividade repelente de mosquitos são plantas aromáticas e ricas em óleos essenciais. Apesar dos produtos derivados de óleos essenciais de plantas apresentarem uma boa eficácia e baixa toxicidade, eles possuem algumas limitações tais como necessidade de padronização química e controle de qualidade e baixo tempo de repelência decorrente da sua elevada volatilidade e baixa estabilidade química. Por essas razões, a utilização de produtos repelentes derivados de óleos essenciais de plantas carece de repetidas aplicações a fim de manter o efeito repelente (Li et al., 2013). Nesse sentido, algumas medidas tecnológicas, tais como a incorporação dos óleos essenciais em formulações de sistemas micro- e nanoemulsionados, têm sido adotadas como uma estratégia para aumentar o tempo de atividade destes metabólitos secundários, melhorando sua estabilidade química e reduzindo a volatilização (Trongtokit et al., 2005).

$\mathrm{O}$ processo de transformação de uma planta medicinal em um medicamento fitoterápico precisa atender às normas estabelecidas para garantir a preservação da integridade química e farmacológica da planta, a fim de que sua ação biológica seja constante e a segurança de uso seja garantida (Han et al., 2019). Sabe-se, ainda, que existem algumas dificuldades inerentes ao desenvolvimento desses medicamentos, em relação à sua própria origem, devido à complexidade dos constituintes da matéria-prima vegetal e a variabilidade na qualidade dos produtos obtidos a partir das mesmas espécies de plantas. Essas características estão relacionadas aos fatores e condições do local de cultivo, ao procedimento de coleta, manuseio e processamento da matéria-prima vegetal. Dessa forma, os produtos vegetais apresentarão variações aumentando ainda mais a necessidade de padronização para garantir eficácia, segurança e qualidade (Liu, Guo, \& Liu, 2018).

As Diretrizes da Organização Mundial de Saúde para teste de eficácia de repelentes de mosquitos em pele humana (2009), recomenda que o método de escolha seja realizado em humanos, uma vez que produz resultados que são relevantes para as condições de uso. Os produtos repelentes precisam garantir uma boa qualidade contendo níveis consistentes de compostos ativos e submetidos a um rigoroso controle. A análise do perfil químico é fundamental, pois o uso de técnicas adequadas pode fornecer um relato preciso de seus constituintes, além de fornecer informações qualitativas e quantitativas (Folashade, Omoregie, \& Ochogu, 2012).

\section{Conclusão}

A partir do desenvolvimento deste protocolo, objetiva-se desenvolver Revisões Sistemáticas para avaliar a qualidade dos estudos de plantas medicinais com atividade repelente de mosquitos e verificar se os produtos utilizados nesses estudos 
estão de acordo com a legislação vigente sobre padronização e controle de qualidade, sabendo que esses fatores são de grande importância para garantir a eficácia do produto repelente.

A presente proposta de revisão sistemática tem relevância perante a necessidade e procura por novos repelentes padronizados, eficazes e seguros no combate a doenças transmitidas por mosquitos. Os pesquisadores buscarão referências que permitam a realização da revisão sistemática para que seja possível formar, por meio desta, forneceremos o caminho metodológico para a realização de Revisões Sistemáticas com evidência científica robusta e confiável.

\section{Agradecimentos}

Os autores agradecem à CAPES - Coordenação de Aperfeiçoamento de Pessoal de Nível Superior, do Ministério da Educação do Brasil. E ao Núcleo de Pesquisa em Produtos Naturais e Assistência Farmacêutica da Universidade Federal de Sergipe, pelo desenvolvimento do presente trabalho.

\section{Referências}

Ali, M. S., Ravikumar, S., \& Beula, J. M. (2012). Bioactivity of seagrass against the dengue fever mosquito Aedes aegypti larvae. Asian Pacific Journal of Tropical Biomedicine, 2(7), 570-573. https://doi.org/10.1016/S2221-1691(12)60099-9

Ali, S., Gugliemini, O., Harber, S., Harrison, A., Houle, L., Ivory, J., et al. (2017) Environmental and Social Change Drive the Explosive Emergence of Zika Virus in the Americas. PLoS Negl Trop Dis 11 (2): e0005135. 10.1371/journal.pntd.0005135.

Brozek, J. L., Akl, E. A., Alonso-Coello, P., Lang, D., Jaeschke, R., Williams, J. W., Phillips, B., Lelgemann, M., Lethaby, A., Bousquet, J., Guyatt, G. H., Schünemann, H. J., \& GRADE Working Group (2009). Grading quality of evidence and strength of recommendations in clinical practice guidelines. Part 1 of 3. An overview of the GRADE approach and grading quality of evidence about interventions. Allergy, 64(5), 669-677. https://doi.org/10.1111/j.13989995.2009.01973.x

Folashade, O., Omoregie, H., \& Ochogu, P. (2012). Standardization of herbal medicines-A review. International Journal of Biodiversity and Conservation, 4(3), 101-112. https://doi.org/10.5897/IJBC11.163

Geetha, R. V., \& Roy, A. (2014). Essential oil repellents-a short review. International Journal of Drug Development and Research, 6(2), $20-27$.

Guyatt, G. H., Oxman, A. D., Vist, G. E., Kunz, R., Falck-Ytter, Y., Alonso-Coello, P., Schünemann, H. J., \& GRADE Working Group (2008). GRADE: an emerging consensus on rating quality of evidence and strength of recommendations. BMJ (Clinical research ed.), 336(7650), 924-926. https://doi.org/10.1136/bmj.39489.470347.AD

Han, L., Wang, R., Zhang, X., Yu, X., Zhou, L., Song, T., \& Bai, C. (2019). Advances in processing and quality control of traditional chinese medicine Coptidis rhizoma (Huanglian): A Review. Journal of AOAC International, 102(3), 699-707. https://doi.org/10.5740/jaoacint.18-0303

Higgins, J. P., Altman, D. G., Gøtzsche, P. C., Jüni, P., Moher, D., Oxman, A. D., Savovic, J., Schulz, K. F., Weeks, L., Sterne, J. A., Cochrane Bias Methods Group, \& Cochrane Statistical Methods Group (2011). The Cochrane Collaboration's tool for assessing risk of bias in randomised trials. BMJ (Clinical research ed.), 343, d5928. https://doi.org/10.1136/bmj.d5928

Li, Y., Kong, D., Huang, R., Liang, H., Xu, C., \& Wu, H. (2013). Variations in essential oil yields and compositions of Cinnamomum cassia leaves at different developmental stages. Industrial Crops and Products, 47, 92-101. 10.1016/j.indcrop.2013.02.031

Liu, C., Guo, D. A., \& Liu, L. (2018). Quality transitivity and traceability system of herbal medicine products based on quality markers. Phytomedicine, 44, 247-257. https://doi.org/10.1016/j.phymed.2018.03.006

McHugh M. L. (2012). Interrater reliability: the kappa statistic. Biochemia medica, 22(3), 276-282.

Moher, D., Shamseer, L., Clarke, M., Ghersi, D., Liberati, A., Petticrew, M., Shekelle, P., Stewart, L. A., \& PRISMA-P Group (2015). Preferred reporting items for systematic review and meta-analysis protocols (PRISMA-P) 2015 statement. Systematic reviews, 4(1), 1. https://doi.org/10.1186/2046-4053-4-1

Ouzzani M., Hammady H., Fedorowicz Z., \& Elmagarmid A. (2016). Rayyan-a Web and mobile App for Systematic Reviews. Syst. Rev. 5 (1), 210.10.1186/s13643-016-0384-4

Page, M. J., McKenzie, J. E., Bossuyt, P. M., Boutron, I., Hoffmann, T. C., Mulrow, C. D., Shamseer, L., Tetzlaff, J. M., Akl, E. A., Brennan, S. E., Chou, R., Glanville, J., Grimshaw, J. M., Hróbjartsson, A., Lalu, M. M., Li, T., Loder, E. W., Mayo-Wilson, E., McDonald, S., McGuinness, L. A., \& Moher, D. (2021). The PRISMA 2020 statement: an updated guideline for reporting systematic reviews. BMJ (Clinical research ed.), 372, n71. https://doi.org/10.1136/bmj.n71

Rueda, L. M (2008). Global diversity of mosquitoes (Insecta: Diptera: Culicidae) in freshwater. Hydrobiology, 595, 477-487. https://doi.org/10.1007/s10750007-9037-x

Trongtokit, Y., Rongsriyam, Y., Komalamisra, N., \& Apiwathnasorn, C. (2005). Comparative repellency of 38 essential oils against mosquito bites. Phytotherapy Research: An International Journal Devoted to Pharmacological and Toxicological Evaluation of Natural Product Derivatives, 19(4), 303-309. https://doi.org/10.1002/ptr.1637 
Research, Society and Development, v. 10, n. 13, e347101321568, 2021

(CC BY 4.0) | ISSN 2525-3409 | DOI: http://dx.doi.org/10.33448/rsd-v10i13.21568

Wilke, A. B. B., \& Marrelli, M. T. (2015). Paratransgenesis: a promising new strategy for mosquito vector control. Parasites \& Vectors, 8(1), 342, 1-9. https://doi.org/10.1186/s13071-015-0959-2

Geetha, R. V., \& Roy, A. (2014). Essential oil repellents-a short review. International Journal of Drug Development and Research, 6(2), 20-27.

George, D., Finn, R. Graham, K. \& Sparagano, O. (2014). Present and future potential of plant-derived products to control arthropods of veterinary and medical significance. Parasites and Vectors. 7. 10.1186/1756-3305-7-28.

Ghosh, A., Chowdhury, N., \& Chandra, G. (2012). Plant extracts as potential mosquito larvicides. The Indian journal of medical research, 135(5), 581-598.

Powell, J. (2018). Mosquito-Borne Human Viral Diseases: Why Aedes aegypti? The American Journal of Tropical Medicine and Hygiene. 98. 10.4269/ajtmh.17-0866.

Pohlit, A. M., Lopes, N. P., Gama, R. A., Tadei, W. P., \& Andrade Neto, V. F. D. (2011). Patent literature on mosquito repellent inventions which contain plant essential oils-a review. 77(6), 598-617. http://dx.doi.org/10.1055/s-0030-1270723.

Tandina, F., Doumbo, O., Yaro, A. S., Traoré, S. F., Parola, P., \& Robert, V. Mosquitoes (Diptera: Culicidae) and mosquito-borne diseases in Mali, West Africa. Parasit Vectors. 11(1):467. 10.1186/s13071-018-3045-8. 